\title{
Calculation of the Magnetic Systems of Diagnostic and Cleaning Pigs for Oil and Gas Pipelines
}

\author{
Alexander LUKHVICH, Andrey LUKYANOV, Alexander POLONEVICH, Vasiliy \\ CHURILO, Institute of Applied Physics of National Academy of Sciences of Belarus, \\ Minsk, Belarus
}

\begin{abstract}
The sources, considered in the paper, of the permanent magnetic field have an axis of symmetry and plane of mirror reflection. Such symmetry usually have magnetic systems of clearing and diagnostic pigs in pipeline transport. The magnetic field in free space environmental the source, can be described by scalar potential satisfying to the Laplace equation. The conclusions about distribution of the magnetic field are made of the common solving of this equation at the account of symmetry of the source. It is shown, that the reduction of sizes of the magnetic system in an axial direction promotes faster recession of intensity of the field with distance and increase of heterogeneity of its distribution on azimuthally angle. The growth of this heterogeneity is promoted also by influence of the ferromagnetic pipe environmental the magnetic system. The conclusion about existence of the optimum order of symmetry of the magnetic system is made which provides its best force characteristics at the given mass of the magnetic material spent on its manufacturing.
\end{abstract}

\section{Contents}

Creation of sources of the magnetic fields providing given spatial distribution and force characteristics (the module of field and his gradient) to set of technical appendices, is significant difficulties in the course of modeling, numerical calculations and experimental researches. At the same time, taking into account only symmetry of a source allows to obtain analytically certain characteristics of the field, without numerical calculations.

Let's consider that on the example of creation of the diagnostic magnetic systems and cleaning pigs for oil and gas pipelines. Although the requirements for functional capacities of these two types of pigs differ somewhat, the design of their magnetic systems is virtually the same. The magnetic system of a pig is shown schematically in Fig. 1. It has the shape of a circular belt consisting of permanent magnets that are located at the same distance from the system's axis (corresponding to the $z$ axis in the figure). Each magnet has the form of a parallelepiped with sides equal to $l, h$ and $d$. Magnetization of each magnet, which is parallel to its side $h$, may be directed either toward the system's axis or in the opposite direction. In all cases, the magnetic system is assumed to be oriented with respect to the $x, y$ and $z$ axes, as shown in the figure. Besides Cartesian coordinates, spherical coordinates $\rho, \vartheta$ and $\varphi$ will be used. They are related to the Cartesian coordinates by the formulas

$$
x=\rho \sin \vartheta \cos \varphi, y=\rho \sin \vartheta \sin \varphi, z=\rho \cos \vartheta .
$$

It is convenient to represent the field distribution in the plane $z=0$ in polar coordinates $r$ and $\varphi$ that are expressed in terms of $x$ and $y$ as

$$
x=r \cos \varphi, y=r \sin \varphi .
$$




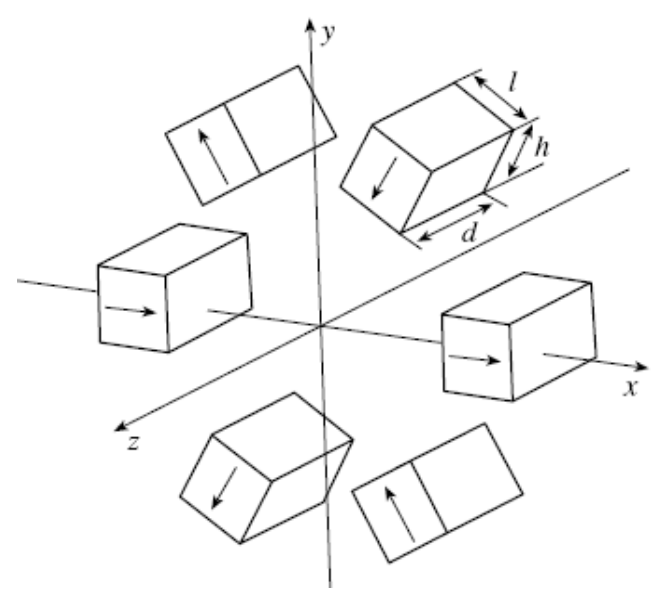

Fig. 1. Schematic layout of magnetic blocks in a pig's system.

The system has a symmetry axis of some order $m$, i.e., it coincides with itself after having been rotated by an angle of $2 \pi / \mathrm{m}$. The figure corresponds to the case of $m=3$.

The distribution of magnetic field strength $\mathbf{H}$ in the free space above the blocks, which contain no field sources, is most important for pigs. In this case, the field is the gradient of scalar potential $\Phi$ satisfying Laplace's equation [1]. The general solution of Laplace's equation is well known. Let us represent it in the spherical coordinate system keeping in mind the system orientation according to fig. 1 [2].

$$
\hat{\mathrm{O}}=\sum_{n=0}^{\infty} \sum_{m=0}^{n} \frac{A_{n m}}{\rho^{n+1}} P_{n}^{m}(\cos \vartheta) \cos m \varphi .
$$

In eqs. (1) $n, m$ are nonnegative integers, and symbol $P_{n}^{m}$ denotes the associated Legendre functions [2]. The specific form of the solution depends on the values of coefficients $A_{n m}$.

The magnetic system is symmetric with respect to plane $z=0$, so that

$$
\hat{\mathrm{O}}(\rho, \vartheta, \varphi)=\hat{\mathrm{O}}(\rho, \pi-\vartheta, \varphi) \text {. }
$$

This requirement is satisfied if the difference $(n-m)$ is an even number [2].

If the magnetic system is characterized by a specific order, the factor in front of $\varphi$ in formula (1) besides being a nonnegative integer, should also be a multiple of the system's order. Therefore, in all cases $n \geq m$.

The terms in the series (1) are usually called the harmonics of order $m$ and degree $n$. Formula (1) shows that this potential is inversely proportional to $\rho^{n+1}$, i.e., the potential diminishes when the distance grows. Since, in all cases, $n \geq m$, this observation allows the conclusion that, with a larger value of order $m$ of a spherical harmonic, the more rapidly this harmonic fades out when the distance increases. Therefore, at sufficiently large distance $\rho$ potential $\hat{O}$ will be determined by the main harmonic, i.e., by the harmonic whose order is the lowest among all harmonics that have nonzero coefficients $A_{n m}$. It is important to consider how the field of a single spherical harmonic behaves.

$$
\Phi=\frac{A_{n m}}{\rho^{n+1}} P_{n}^{m}(\cos \vartheta) \cos m \varphi .
$$

To obtain the field distribution in plane $z=0$ it is sufficient to substitute $\vartheta=\frac{\pi}{2}$ into formula (2) . Component $H_{\vartheta}$ then vanishes, and the two remaining components may be represented easily in terms of polar coordinates $r$ and $\varphi$ :

$$
\begin{gathered}
H_{r}=A_{n m} \frac{(n+1)}{r^{n+2}} P_{n}^{m}(0) \cos m \varphi, \\
H_{\varphi}=A_{n m} \frac{m}{r^{n+2}} P_{n}^{m}(0) \sin m \varphi .
\end{gathered}
$$

These formulas show that the field of a harmonic of order $m$ decreases with the growth of distance as $\frac{1}{r^{n+2}}$, where $n \geq m$. In addition, analysis of these expressions shows that the factor in front of the cosine function in (3) and the sine function in (4) are not the 
same. If the cosine amplitude in (3) is denoted as $A_{r}$, and the sine amplitude in (4) is designated as $A_{\varphi}$, their ratio may be represented as:

$$
\frac{A_{\varphi}}{A_{r}}=\frac{m}{n+1}<1 .
$$

This expression shows that the absolute value of the field for fixed $r$ depends on $\varphi$ and attains a minimum when $\cos m \varphi=0$, and $\sin m \varphi=1$, i.e.,

$$
\begin{aligned}
m \varphi & =\frac{\pi}{2}, \\
\text { thus yielding } \quad \varphi_{\min } & =\frac{\pi}{2 m} .
\end{aligned}
$$

This angle corresponds to the center of the interval between two adjacent magnets. In the case of $m=3$ (see Fig. 1),

$$
\varphi_{\min }=\frac{\pi}{2 \cdot 3}=30^{\circ} .
$$

The magnetic systems of cleaning pigs should be compared on the basis of their force of impact. Since the latter depends not only on the magnetic system itself but also on the magnetic properties of an attracted item, the best approximation is an induced magnetic dipole whose magnetic moment is proportional to the field strength. The force acting on an induced dipole in the direction of $r$ is known [1] to be proportional to the product of the field's absolute value and its gradient in this direction

$$
F_{r} \sim H \frac{\partial H}{\partial r} \text {. }
$$

Hereinafter, symbol $H$ without sub- and superscripts denotes the absolute value of the magnetic field's strength vector. Having substituted the field with components (3) and (4) into formula (6), we arrive at

$$
F_{r} \sim-\left[A_{n m} P_{n}^{m}(0)\right]^{2} \cdot \frac{n+2}{r^{2 n+5}}\left[m^{2}+\left(n^{2}+2 n-m^{2}+1\right) \cos ^{2} m \varphi\right] .
$$

The force depends on $\varphi$, attaining the maximum value when the cosine is \pm 1 and the minimum value when the cosine vanishes, i.e., coincides with minimum of $H(5)$. Therefore, the force's maxima correspond to the centers of the magnets, while the minima correspond to the centers of the intervals between the magnets.

In paper [3] us it is shown, that if the dimension $d$ of the magnetic system in the direction of the pipe's axis diminish, harmonics with greater degrees $n$ in harmonious structure of its field increases. Formulas (3), (4), and (7) show that it promotes faster recession of a field and force with radius $r$. At the same time, the amplitude oscillation of forces increases with change of an angle $\varphi$ as the factor in front of the cosine function in the formula (7) contains the component $n^{2}+2 n$.

Let us assume for problem simplification that the dimension $d$ of the magnetic system so large, that it is possible to consider the field independent on $z$. Then it is convenient to describe the problem in polar coordinates $r$ and $\varphi$. Inside of a pipe over a cylindrical surface of radius $r_{0}$ the superficial density $\sigma$ of a magnetic charge is distributed

$$
\sigma=\sigma_{0} \cos m \varphi
$$

where $\sigma_{0}$ is the amplitude value of the charge density. We shall accept the distribution (8) as a mathematical model of magnetic system. The field of the source (8) is easy to obtain from the common solution of Laplace's equation in polar coordinates [4].

The field in the external area $\left(r>r_{0}\right)$ has the form: 


$$
H_{r}^{e}(\sigma)=\frac{\sigma_{0}}{2}\left(\frac{r_{0}}{r}\right)^{m+1} \cos m \varphi ; H_{\varphi}^{e}(\sigma)=\frac{\sigma_{0}}{2}\left(\frac{r_{0}}{r}\right)^{m+1} \sin m \varphi .
$$

In the internal area $\left(r<r_{0}\right)$ :

$$
H_{r}^{i}(\sigma)=-\frac{\sigma_{0}}{2}\left(\frac{r_{0}}{r}\right)^{m-1} \cos m \varphi ; H_{\varphi}^{i}(\sigma)=\frac{\sigma_{0}}{2}\left(\frac{r_{0}}{r}\right)^{m-1} \sin m \varphi .
$$

The cross section of the pipe containing the source in its interior is shown in Fig. 2. The field in spatial areas I $\left(r_{0}<r<r_{1}\right)$, II $\left(r_{1}<r<r_{2}\right)$ and III $\left(r>r_{2}\right)$ is a sum of the primary field (9) and the secondary field of the pipe magnetized by the primary field. Since the pipe's material contained in area II is considered a linear magnetic material, the following relation holds in this area:

$$
\operatorname{div} \mathbf{M}=0,
$$

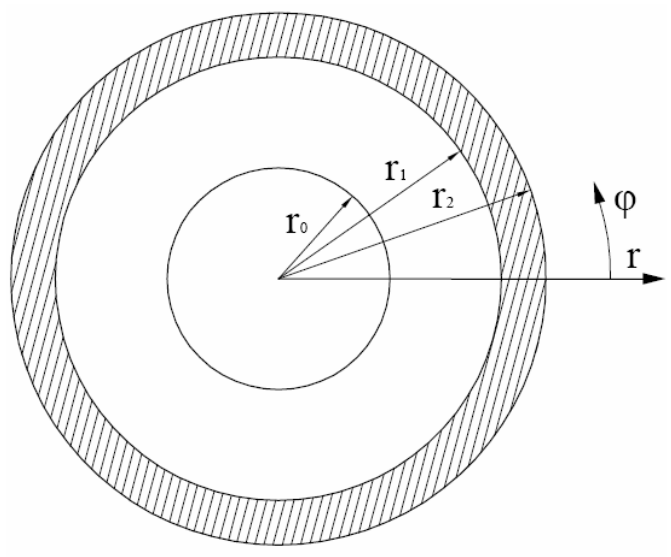

Fig. 2. Cross section of a pipe containing a field source.

i.e., this area is free from the volume charge density. This means that only surface charge densities $\sigma_{1}$ and $\sigma_{2}$, distributed over surfaces $r=r_{1}$ and $r=r_{2}$, respectively, may be the secondary sources of the field [1]. Moreover, since the pipe's material has linear properties and owing to the symmetry of the problem, distributions $\sigma_{1}$ and $\sigma_{2}$ have the same degree $m$, as primary source (8):

$$
\begin{aligned}
& \sigma_{1}=\sigma_{01} \cos m \varphi, \\
& \sigma_{2}=\sigma_{02} \cos m \varphi .
\end{aligned}
$$

Here, $\sigma_{01}$ and $\sigma_{02}$ are unknown amplitudes that may be expressed in terms of the magnetic system's parameters $\mu, \sigma_{0}, r_{0}, r_{1}, r_{2}$. To do so, let us derive expressions for the field components in areas I, II, and III.

Area $I$ is external with respect to charge density $\sigma$ and internal with respect to densities $\sigma_{1}$ and $\sigma_{2}$. Therefore,

$$
\begin{aligned}
& H_{r}^{\mathrm{I}}=H_{r}^{e}(\sigma)+H_{r}^{i}\left(\sigma_{1}\right)+H_{r}^{i}\left(\sigma_{2}\right) ; \\
& H_{\varphi}^{\mathrm{I}}=H_{\varphi}^{e}(\sigma)+H_{\varphi}^{i}\left(\sigma_{1}\right)+H_{\varphi}^{i}\left(\sigma_{2}\right) .
\end{aligned}
$$

Distributions of charge densities $\sigma_{1}$ and $\sigma_{2}$ are governed by discontinuities of normal component $M_{r}$ of the magnetization on surfaces $r=r_{1}$ and $r=r_{2}$ respectively:

$$
\begin{gathered}
\sigma_{1}(\varphi)=\sigma_{01} \cos m \varphi=-M_{r}^{\mathrm{II}}\left(r_{1}, \varphi\right) ; \\
\sigma_{2}(\varphi)=\sigma_{02} \cos m \varphi=M_{r}^{\mathrm{II}}\left(r_{2}, \varphi\right) .
\end{gathered}
$$


Using expressions (14) and (15) and taking into account (8), (12) and (13), as well as (9) and (10), we arrive at:

$$
\begin{aligned}
& H_{r}^{\mathrm{I}}=\frac{\sigma_{0}}{2}\left(\frac{r_{0}}{r}\right)^{m+1}\left(1+b r^{2 m}\right) \cos m \varphi, \\
& H_{\varphi}^{\mathrm{I}}=\frac{\sigma_{0}}{2}\left(\frac{r_{0}}{r}\right)^{m+1}\left(1-b r^{2 m}\right) \sin m \varphi,
\end{aligned}
$$

where the following notation was introduced for convenience:

$$
b=\frac{(\mu-1)(\mu+1)\left(r_{2}^{2 m}-r_{1}^{2 m}\right)}{r_{1}^{2 m}\left[(\mu+1)^{2} r_{2}^{2 m}-(\mu-1)^{2} r_{1}^{2 m}\right]} \text {. }
$$

The value of $b$ is determined only by pipe parameters $\mu, r_{1}$ and $r_{2}$ and degree $m$ of the primary field's source.

Let us estimate the field in area I, by its effect on an induced dipole alone, i.e., according to formula (6).

If $\mu>1$, the value of $b$ given by the formula is not zero and, according to expressions (18 and (19), the absolute value of the field is

$$
H=\frac{\sigma_{0}}{2}\left(\frac{r_{0}}{r}\right)^{m+1}\left[1+b^{2} r^{4 m}+2 b r^{2 m} \cos (2 m \varphi)\right]^{\frac{1}{2}},
$$

thus yielding the following expression for the force component:

$$
F_{r} \sim H \frac{\partial H}{\partial r}=\frac{(m+1) \sigma_{0}^{2} r_{0}^{2(m+1)}}{4 r^{(2 m+3)}}\left[-1+\frac{(m-1) b^{2}}{m+1} r^{4 m}-\frac{2 b}{m+1} r^{2 m} \cos (2 m \varphi)\right] .
$$

The factor in front of the square brackets is the absolute value of the force in the absence of the pipe $(\mu=1)$. Analysis of the dependence of the dimensionless quantity in square brackets on angle $\varphi$ yields the increment of the force induced by the pipe as compared to the force in the absence of the pipe, which is adopted to be -1 . Since formula (20) is cumbersome, we have set specific parameters of a pipe $\left(r_{1}=0,59 \mathrm{~m}\right.$ and $\left.r_{2}=0,6 \mathrm{~m}\right)$ and the magnetic system's degree $m=3$ and calculated some values of $b$ for several values of permeability $\mu$ of the pipe's material. The results obtained are presented below.

$$
\begin{array}{cccccc}
\mu \ldots . . . & 1 & 5 & 50 & 500 & \infty \\
b . \ldots . & 0 & 2.534 & 13.21 & 21.96 & 23.71
\end{array}
$$

The expression in the square brackets in formulas (20) for $m=3$ adopt the form

$$
F_{r} \sim-1+\frac{1}{2} b^{2} r^{12}-\frac{1}{2} b r^{6} \cos 6 \varphi \text {. }
$$




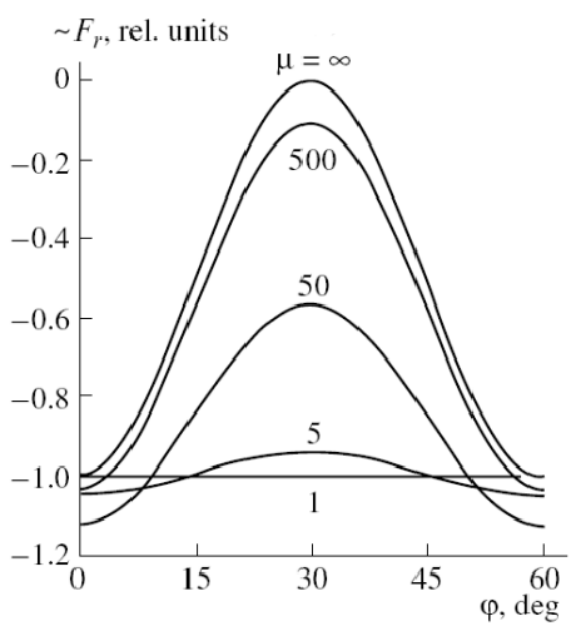

Fig. 3. Angular dependence of the force on the pipe's internal surface for radial components.
Figure 3 display dependences (22) plotted for several values of permeability $\mu$ for $r=r_{1}=0,59 \mathrm{~m}$, i.e., on the surface of the pipe itself. Figure, shows that, while for $\mu=1$ the normal component of the force does not depend on $\varphi$ and equals -1, when permeability increases, the force oscillations emerge. The period of oscillations is $60^{\circ}$, and their amplitude increases as the permeability grows and attains maximum at $\mu=\infty$. In this limiting case, the force ranges from -1 to 0 .

The parameters of field source (9) $\left(r_{0}, \sigma\right.$ and $\sigma_{0}$ ) may be considered as kinds of prototypes or analogues of a real system's characteristics: namely, $r_{0}$ is an analogue of the system's dimension in the radial direction; $\sigma_{0}$ is an analogue

of the properties of the materials from which permanent magnets are made; and charge density $\sigma$ (its absolute value), being integrated over a circle with radius $r_{0}$, yields a total charge and thus is an analogue of the amount of the magnetic material used to make such a magnetic system.

As follows from formula (19),

$$
\int_{0}^{2 \pi}|\sigma| r_{0} d \varphi=\int_{0}^{2 \pi} \sigma_{0}|\cos m \varphi| r_{0} d \varphi=4 \sigma_{0} r_{0}
$$

i.e., the amount of the material does not depend on system's order $m$. In this case, it is natural to determine what order $m$ should be for the given amount of material in order to ensure the strongest attraction. To do so, it is necessary to find the extremum of expression (22) over $m$ for $b=0$. Assuming that $m$ is a continuous variable, we set the derivative of expression (22) over $m$ equal to zero. Then, the optimum order is

$$
m_{0}=\frac{1}{2 \ln r / r_{0}}-1 \text {. }
$$

The system's optimum order $m_{0}$ depends on the ratio $r / r_{0}$. In other words, the larger the pipe's radius as compared to $r_{0}$, the smaller the system's order should be.

The block design magnetic systems of cleaning pigs, reduction of the sizes of blocks, as well as material of pipelines lead to angular dependence of their force characteristics. The dependences obtained in the work allow to perform estimation of this influence by the design optimization.

\section{References}

1. Tamm, I.E., Osnovy teorii elektrichestva (Foundations of the Theory of Electricity), Moscow: Nauka, 1976.

2. Ango, A., Mathematics for Electro- and Radioengineers. Translated under the title Matematika dlya elektro- i radioinzhenerov, Moscow: Nauka, 1965.

3. Lukhvich A. A., Polonevich A. A., Churilo V. R., Raschet Magnitnyh Sistem Diagnosticheskih I Ochistnyh Porshneiy Gazo- I Nefteprovodov, 2006 г, vol.I, pp. 26-36 4. Smirnov, V.I., Kurs vysshei matematiki (Course of Higher Mathematics), Moscow: Nauka, 1974, vol. II. 